\title{
Treatment of advanced stage classical Hodgkin lymphoma: a pathway through the maze
}

\author{
Hildyard C AT*, Vora SM and Collins GP \\ Department of Clinical Haematology, Oxford Cancer and Haematology Centre, Churchill Hospital, Oxford OX3 7LE, UK
}

\begin{abstract}
The wealth of trial data, complex trial design, and variation in treatment standards in advanced stage classical Hodgkin lymphoma can be difficult to navigate when deciding on the best treatment for patients. In this review, we appraise and synthesise this evidence, in order to explain our suggested treatment approach.

Since the 1960s, cures have been achieved in advanced stage classical Hodgkin lymphoma (cHL) through the use of multi-agent chemotherapy regimens often with addition of radiotherapy. Since then, treatment has been improved through a process of rigorous testing in clinical trials, such that progression free survival (PFS) and overall survival rates (OS) are now excellent, especially in younger patients. More recently there has been a shift in focus towards minimizing toxicity (both short and long term) without compromising efficacy. Recent trials have tried to achieve this by risk-stratifying patients, both at baseline and according to response to treatment, so as to guide escalation or de-escalation of therapy. Uncertainty as to where this equipoise between efficacy and toxicity lies has also led to two international standards for treatment of cHL: ABVD (doxorubicin, bleomycin, vinblastine, dacarbazine) and escalated BEACOPP (bleomycin, etoposide, doxorubicin, cyclophosphamide, vincristine, procarbazine, prednisolone). It has also influenced decisions on combined modality treatment with radiotherapy.
\end{abstract}

The wealth of trial data, complex trial design, and variation in treatment standards can be difficult to navigate when deciding on the best treatment for our patients. In this article, we aim to provide a possible pathway through this maze.

\section{Definition of advanced classical Hodgkin Lymphoma}

Comparison across clinical trials is always difficult. However, the lack of a standardized definition of "advanced stage" cHL is particularly problematic in this context. Some trials limit "advanced stage" to stage III-IV disease (using the Ann-Arbor staging system), while other trials also include stage IIB with bulky nodal disease $(>10 \mathrm{~cm}$ or $>33 \%$ of the trans-thoracic diameter). Furthermore, trials have historically used CT to stage patients whereas more recent studies have incorporated FDG$\mathrm{PET} / \mathrm{CT}$ as standard.

\section{Efficacy of ABVD versus escBEACOPP}

The German HD9 trial [1] first demonstrated improved FFTF (freedom from treatment failure), and with further follow up overall survival (OS) [2], with escalated BEACOPP (escBEACOPP) compared with COPP (cyclophosphamide, vincristine, procarbazine, prednisolone)-ABVD. A major criticism of the trial was that the comparator arm (COPP-ABVD) was not regarded as standard of care at the time of trial publication and the outcome of patients in that arm was not as good as expected with modern delivery of ABVD. Since then, multiple trials have compared ABVD with escBEACOPP in order to try to reproduce these results $[3,4]$.

A meta-analysis of these trials, published in 2011 [5] showed an improved PFS but not OS with escBEACOPP. However, an updated meta-analysis published in 2017 [6] also showed a significant improvement in OS. This meta-analysis identified five eligible trials (HD9, HD14, HD2000, GSM-HD and EORTC-20012) that compared at least two cycles of escBEACOPP with four cycles of ABVD, thus ensuring equivalent doses. The meta-analysis excluded trials incorporating standard dose BEACOPP rather than escBEACOPP as this was shown to be inferior in the HD-9 study (1). The hazard ratio
(HR) for treatment with escBEACOPP versus ABVD was 0.74 for OS and 0.54 for PFS. With any meta-analysis however, the results are only as reliable as the trials analysed. The highest weighting within the study was for HD9 (which was given a weight of $35.3 \%$ ) with the attendant issues of the control arm in that trial. Significant benefit of overall survival therefore remains uncertain although, given the consistent improvement in disease control, a degree of OS improvement is likely.

\section{Toxicity of ABVD versus escBEACOPP}

The Cochrane 2017 meta-analysis upheld opinion that escBEACOPP is more toxic than ABVD [6]. There was significantly increased haematological toxicitity as well as some non-haematological toxicities including mucositis, neurological toxicity and respiratory tract infections. There was no significant difference in some other nonhaematological toxicities including constipation, nausea/ vomiting, cardiotoxicity and skin rashes. This increased toxicity did not result in a significantly increased treatment-related mortality (TRM). However, the number of patients dying from treatment is very low and therefore this comparison is based on very small numbers. There was an increased risk of AML/MDS in the escBEACOPP arm, but no difference in the rate of overall secondary malignancies. However, the follow up period may be too short to make a valid comparison as most secondary malignancies would not be expected to occur until approximately 15 years post treatment.

${ }^{*}$ Correspondence to: Hildyard C AT, Department of Clinical Haematology, Oxford Cancer and Haematology Centre, Churchill Hospital, Oxford OX3 7LE, UK, Tel: 07799647985; E-mail: Catherine.Hildyard@ouh.nhs.uk

Received: December 12, 2018; Accepted: December 19, 2018; Published: December 21, 2018 
EscBEACOPP is predicted to be more harmful to fertility, whereas ABVD is considered relatively fertility sparing, especially in women. Comparing data across trials supports this prediction. In the HD-14 trial [7], 100\% of women under the age of 30 and $96 \%$ of women over the age of 30 regained regular menstrual cycles after treatment with 4 cycles of ABVD. By contrast, in the HD- 15 trial [8], 82\% of women less than 30 and only $45 \%$ of women over 30 regained menstrual cycles after 6-8 cycles of escBEACOPP. Furthermore, the UK RATHL trial has recently reported a sub-study looking at ovarian function in a subset of patients [9]. Anti-Mullerian hormone (AMH) levels were initially reduced in both AVBD and escBEACOPP treated patients, but levels recovered in most ABVD treated patients within 1 year whereas little recovery was seen following BEACOPP treated patients at this time point. The impact of different numbers of cycles of each regime is not clear. There appears to be less of a difference between the two regimes for male infertility [10].

\section{Use of interim PET to guide adaptation of therapy}

There is well established evidence that a positive interim PET (PET2) after 2 cycles of chemotherapy, defined by a Deauville score of 4-5 (D 4-5), predicts for inferior PFS and OS compared with a negative PET2, defined by a Deauville score of 1-2 (D1-2) [11]. Different trials vary as to whether a PET2 with a Deauville score of 3 (D3) is classified as positive or negative, with most classifying it as negative.

Several risk-adapted trials have illustrated how PET2 can be used to de-escalate therapy in a cohort of patients who are predicted to have superior outcomes. In the RATHL trial [12], 1214 patients with advanced stage $\mathrm{CHL}$ received 2 cycles of ABVD followed by PET2. In the event of a negative PET2 (D 1-3), patients were randomized to receive either 4 cycles of ABVD or to drop the bleomycin and receive 4 cycles of AVD. There was no significant difference in PFS or OS between the two arms with a significant reduction in pulmonary toxicity in the AVD arm. On the basis of these results, omission of bleomycin in the context of patients treated with ABVD with negative PET2 has become standard of care.

In the HD-18 trial [13] patients with advanced stage cHL were treated with 2 cycles of escBEACOPP followed by PET2. Those with a negative PET2 (D1-2) were randomized between 6 further cycles of escBEACOPP versus 2 further cycles (although part way through the trial the protocol was amended based on results from another trial, so the control arm got 4 extra cycles rather than 6). There were no differences in PFS or OS between the two arms with a significant reduction in toxicity seen in patients receiving a total of 4 cycles compared with 6 or 8 . Furthermore results confirmed the efficacy of this regimen, with $5 y$ PFS rates of just over $90 \%$ for all patients.

The AHL2011 [14] trial treated patients with 2 cycles of escBEACOPP followed by PET2. Patients in the control arm did not receive adapted therapy based on the results of PET2 but instead went on to receive 4 further cycles of escBEACOPP. Patients in the experimental arm with positive PET2 (D4-5, defined by SUV of greater than $140 \%$ of liver background, to improve inter-reader reproducibility) received 4 further cycles of escBEACOPP whereas those with negative PET2 (D1-3 or SUV less than 140\% of liver background) received 4 cycles of ABVD. There was no significant difference in PFS between the standard arm and the experimental arm, $84 \%$ of whom were deescalated to ABVD. Again, results were impressive, although not quite as good as the HD18 trial, with an estimated 4y PFS of $87 \%$ in both arms.

\section{Conclusions from risk-adapted trials}

Baseline characteristics varied considerably in the trials discussed above. For example, the RATHL trial [12] incorporated patients with stage IIA cHL with bulk or $>3$ sites of nodal involvement. Indeed, one third of patients were stage II. There was, however, no upper age limit for enrolment and over $25 \%$ of patients were 45 years of age or older, an age group usually associated with a worse prognosis. HD18 [13] and AHL2011 [14] only included stage IIB-IV patients, and the proportion of stage III-stage IV was much higher, for example $92 \%$ of patients in HD18. Upper age limit for both trials was 60 years of age as escBEACOPP is associated with a high treatment related mortality in older patients. However, several important conclusions can be drawn by comparing and contrasting these trials.

Sub-group analysis of both RATHL [12] and HD-18 [13] demonstrated that patients with Deauville 3 on PET2 have similar prognoses to those with Deauville 1-2, suggesting that it is reasonable to count Deauville 3 as negative.

Despite the large number of stage II patients in the RATHL trial, the outcome for PET2 negative patients was not as good as predicted, with a $3 y$ PFS of $85 \%$ (the trial had initially been powered assuming a $3 y$ PFS of 95\%). Furthermore, PET2 positive patients who were escalated to either escBEACOPP or BEACOPP-14 had a disappointing 3y PFS of $67.5 \%$. This is in contrast to the HD18 trial which treated more patients with advanced stage disease but showed a 3 year PFS of $92.2 \%$ for PET2 negative patients. Even for PET2 positive disease, 3 year PFS was reported as $89.7 \%$. The data was subsequently re-analysed such that D3 patients were removed from the positive PET2 group, but even then the 3year PFS was an impressive 87.6\% [15]. This lends more evidence to an improved PFS with escBEACOPP compared with ABVD. From the RATHL trial, it appears that escalating patients who have a positive PET2 after ABVD does not provide an effective 'rescue' for many. By contrast, the AHL2011 trial supports a de-escalation strategy whereby patients treated with $2 \mathrm{x}$ escBEACOPP who achieve a negative PET2 can be safely de-escalated to $4 \mathrm{x}$ ABVD. The disadvantage to this approach is that all patients are exposed to intensive chemotherapy with attendant toxicities.

The RATHL trial [12] demonstrated that even when PET2 is negative, initial stage and international prognostic score (IPSS) impact on survival. This is an important observation because the majority of relapses actually occur in PET2 negative patients: in the RATHL trial, 1/5.5 PET2 negative patients relapsed. It provides a rationale for risk stratifying treatment at baseline as well as after PET2 results. For example, 3 year PFS for PET2 negative stage II was $88.88 \%$, compared with $80 \%$ for stage IV. Similarly, 3 year PFS was $86.7 \%$ for PET2 negative IPSS $0-2$ and $81.6 \%$ for IPSS $\geq 3$. With longer follow up, an overall survival difference has emerged in RATHL treated patients with $3 y$ OS of 98.6\% for IPSS 0-2 and 95.2\% for IPSS 3 and above [16].

\section{Modifying escBEACOPP to preserve fertility}

An important consideration in the choice between upfront escBEACOPP and ABVD is potential impact on fertility. Most evidence suggests that escBEACOPP is significantly more toxic in this regard [17]. This is of particular concern given that $\mathrm{cHL}$ incidence peaks in the third decade and potentially before a patient has had a chance to start a family. Fertility preservation options are more challenging in women compared with men. While sperm storage is non-invasive and highly successful, egg and embryo storage require hormonal stimulation which may result in a delay to treatment initiation and 
an invasive collection procedure. It is also expensive and not always available. Although much quicker, ovarian tissue cryopreservation is more experimental and also requires a surgical procedure.

There is some evidence that dacarbazine is less gonadotoxic than procarbazine and that this could therefore be substituted without compromising efficacy. Modifying a paediatric protocol [18], OEPACOPDAC (vincristine, etoposide, prednisolone, doxorubicin, cyclophosphamide, vincristine, prednisolone, dacarbazine) was compared with OPPA (vincristine, prednisolone, procarbazine, doxorubicin)- COPP (cyclophosphamide, vincristine, procarbazine, prednisolone), with no significant difference in event free survival (EFS) and less impact in markers of reduced fertility. Interestingly, there was also less haematological toxicity with COPDac compared with COPP. Extrapolating this to adult protocols, it has been suggested that escBEACOPP could be modified to escBEACOPDac. Although there is no published data, many centres have incorporated this change.

\section{Role of radiotherapy in advanced $\mathrm{cHL}$}

Radiotherapy is less well integrated into treatment pathways for advanced stage cHL compared with early stage cHL. There is considerable variation in practice as to whether radiotherapy is given at all and for what indication, whether to sites of original bulk disease ( $>10 \mathrm{~cm}$ or $>1 / 3$ of the intra-thoracic diameter) or to sites of residual FDG-PET avidity. This is reflected in clinical trials, which have focused predominantly on the impact of different chemotherapy regimens and PET2 adapted therapy rather than on the impact of radiotherapy.

The LY09 study [19] compared ABVD with other historical multidrug regimens including ChLVPP/PABLOE and ChLVPP/ EVA. There was a high uptake of radiotherapy in this trial (43\%) although this was not specified in the trial protocol. Interestingly, there was no difference in outcome according to chemotherapy regimen. EFS was superior for patients who received radiotherapy versus those who did not: $86 \%$ versus $71 \%$. Similarly, retrospective analysis of the SEER (Surveillance, epidemiology and end results) database, showed a significant improvement in outcome in patients with advanced stage $\mathrm{cHL}$ who received radiotherapy in addition to chemotherapy: 5year OS $91.6 \%$ versus $71.4 \%$ [20]. Whether this apparent advantage in PFS for radiotherapy applies to the same extent with more modern chemotherapy regimens, especially escBEACOPP, is not clear.

In the HD15 trial [8], which established an improved overall survival with 6 cycles of escBEACOPP compared with 8 cycles of escBEACOPP due to lower treatment toxicity, radiotherapy was given only to PET-avid disease of greater than $2.5 \mathrm{~cm}$ on end of treatment scan. This criterion meant that only $11 \%$ of patients went on to receive radiotherapy with impressive 5-year FFTF rates of $89.3 \%$ and OS of 95.3\%. However, this has not been established as an evidence-based standard of care for patients treated with ABVD and controversy persists as to which patients benefit from radiotherapy, whether all patients with bulk, or just those with persistent FDG avidity on their end of treatment scan. Indeed, the RATHL trial [12] did not mandate radiotherapy and less than $5 \%$ of patients received this treatment modality. Some have argued that this maybe one reason why observed PFS rates were not as high as predicted.

\section{Incorporation of targeted therapies into the front line setting of advanced cHL}

Attempts have recently been made to improve the efficacy of ABVD whilst maintaining its relatively low toxicity rates. For example, in the ECHELON-1 trial [21], six cycles of ABVD were compared with 6 cycles of A-AVD, substituting the conjugated, anti-CD30 antibody Brentuximab Vedotin for bleomycin. The authors reported a significantly improved 2 year modified PFS of $82.1 \%$ with A-AVD versus $77.2 \%$ with $A B V D$. However, the validity of these findings has subsequently been questioned because the authors used a nonstandard end-point, "modified PFS". This was defined as progression or death (as per standard PFS) but also included chemotherapy or radiotherapy given, at the investigators' discretion, for D3-5 residuum on end of treatment PET-CT (ePET). Many would dispute this group as demonstrating true progressive disease without confirmatory biopsy or follow-up scan. This creates the potential for bias as the trial was unblinded, and investigators may therefore have been more likely to give additional treatment to patients on what they perceived as the less potent arm i.e. ABVD. There was significantly increased peripheral neuropathy (including grade 3 ) and neutropenia in the A-AVD arm although reduced pulmonary toxicity compared with ABVD. However, this trial was not PET2 adapted and therefore did not omit bleomycin in the PET2 negative ABVD-treated group. Less pulmonary toxicity is likely to have occurred in the ABVD arm had this occurred.

Rituximab has also been trialed as a targeted therapy that might improve efficacy in combination with chemotherapy in cHL. Hodgkin and Reed-Sternberg (HRS) cells express CD20 in 20-30\% cases, as do $\mathrm{B}$-lymphocytes in the surrounding inflammatory microenvironment [22]. However, in a randomized phase II trial, the addition of Rituximab to ABVD did not improve PFS compared with ABVD [22]. Similarly, the addition of Rituximab to escBEACOPP for patients with positive PET2 in the HD18 trial did not improve PFS [13].

\section{Incorporation of immunotherapy agents into the frontline setting of advanced $\mathrm{cHL}$}

Immune checkpoint inhibitors targeting the PD-1/ PD-L1 and PDL2 pathway have shown excellent responses in relapsed/ refractory cHL [24,25]. Genetic alterations leading to overexpression of programmed cell death ligands $\mathrm{PD}-\mathrm{L} 1$ and $\mathrm{PD}-\mathrm{L} 2$ are characteristic in cHL [26], making it a particularly sensitive tumour sub-type. Their use is less well established in frontline treatment. However, there is some preliminary data showing efficacy prior to, and in combination with conventional chemotherapy.

While cohorts A, B, and C in the CheckMate 205 trial assessed the efficacy of the PD-1 inhibitor nivolumab in relapsed cHL postautologous stem cell transplant, cohort D assessed efficacy of nivolumab monotherapy followed by nivolumab- AVD combination therapy in newly diagnosed stage IIB-IV cHL $[24,27]$. The trial was very small and was designed to investigate safety. However the overall response rate (ORR) was $84 \%$ with a complete response rate (CR) of $67 \% .9$ month PFS was $94 \%$. Toxicity was not significantly in excess of that seen with ABVD chemotherapy. Further evaluation of these agents is warranted.

\section{Advanced stage HL in the elderly}

A sub-group worthy of particular mention is advanced stage cHL occurring in patients over the age of 60 years. Elderly patients account for $20-30 \%$ of HL patients and often present with high-risk features. They have inferior disease outcomes compared with younger patients [28]

EscBEACOPP is particularly poorly tolerated in this group. Following the finding of a $17.2 \%$ mortality rate with this regime in the 60-65 year age group in the HD-12 trial [29] (compared with $2 \%$ in those less than 60), the German Hodgkin Lymphoma Study 
Group (GHSG) suggested an upper age limit of 60 for treatment with escBEACOPP [29].

ABVD is somewhat better tolerated in this group but is still associated with increased toxicity compared with in younger patients and treatment attenuation is common [30]. In a sub-group analysis of patients over the age of 60 from the HD10 and HD11 trials, treatment was terminated early in $8-18 \%$, with $68 \%$ of patients experiencing grade III/IV toxicities with 4 cycles of ABVD. Dose attenuation and treatment delay also contribute to inferior PFS rates: 5 year PFS was $79 \%$ in patients over the age of 60 years in HD10 compared with $96 \%$ in younger patients and $69 \%$ compared with $86 \%$ in HD11.Bleomycin lung toxicity in particular is more common in older patients [31] and related mortality is high in elderly patients. Patients over 60 from the GHSG HD10 and HD13 trials receiving 4 cycles of ABVD had significantly higher rates of bleomycin lung toxicity (10\%) and 3 of 7 cases were lethal [32]. However, 2 cycles of either ABVD or AVD had similarly low rates of respiratory complications and grade III or IV toxicities, without affecting PFS or OS. 2 cycles of ABVD is recommended as a maximum in older patients with de-escalation to AVD thereafter.

Other alternative regimes have been trialed in the elderly population. These include CHOP (cyclophosphamide, doxorubicin, vincristine, prednisolone) [33] VEPEMB (vinblastine, cyclophosphadmide, procarbazine, prednisolone, etoposide, mitoxantrone, bleomycin) [34] PVAG (prednisolone, vinblastine, doxorubicin, gemcitabine) [35] and ChlVPP (chlorambucil, vinblastine, procarbazine and prednisolone) [36]. However, all were associated with disappointing progression free survival rates, when compared with ABVD, although this may partially reflect the frailty of the study populations.

Use of targeted agents to reduce the need for chemotherapy may be an attractive option for elderly patients in the future. In a phase II trial of Brentuximab Vedotin monotherapy in patients over the age of 60 with cHL, $52 \%$ of whom were ineligible for chemotherapy due to poor performance status or comorbidities, ORR was $92 \%$ and CR 73\% [37]. While these preliminary data are promising, toxicity was significant with $78 \%$ of patients experiencing peripheral neuropathy and remissions were generally short. Likewise, there is an interest in using PD-1/ PD-L1 inhibitors as monotherapy upfront in patients not fit enough for conventional chemotherapy. An upcoming phase II, Australian trial (PLIMATH) [38] has been designed to investigate this.

\section{A possible pathway through the maze}

The proposal we are suggesting here takes into account a number of factors:

- EscBEACOPP is a more effective but significantly more toxic chemotherapy regimen compared with ABVD and should therefore be reserved for the highest risk patients who have a worse OS with ABVD.

- EscBEACOPDac is likely to be equivalent in efficacy to escBEACOPP and is potentially more fertility sparing and predicted to have less haematological toxicity.

- Poor risk patients when treated with an ABVD approach can be identified by high baseline IPSS as well as positive PET2.

- A negative PET2 scan can be used to de-escalate treatment.

- Combined modality treatment with radiotherapy is likely to enhance efficacy but with increased risk of long-term sequelae. Patients with positive ePET or those with initial bulk may derive most benefit from the addition of radiotherapy.
- Treatment should be personalized to the patients' individual comorbidities and preferences as much as possible.

- EscBEACOPP/ escBEACOPDac are likely to be too toxic in the $>60$ s age group.

On this basis, for low IPSS (0-2) patients it seems reasonable to start with 2x ABVD and follow a RATHL-style PET adapted process whereby patients with a negative (D1-3) PET2 are de-escalated to $4 \mathrm{x}$ AVD and those with a positive PET2 (D4-5) are escalated to $4 \mathrm{x}$ escBEACOPDac. On the other hand, for high risk IPSS $(\geq 3)$ patients, $2 \mathrm{x}$ escBEACOPDac may be a more appropriate initial treatment with de-escalation following a negative PET2 to either $2 \times$ further escBEACOPDac (following an HD18 protocol) or 4x ABVD (following an AHL2011 protocol - it would also seem reasonable to omit bleomycin in this context although in AHL2011 full ABVD was given). For high IPSS patients with positive PET2 we suggest $4 \mathrm{x}$ further escBEACOPDac (as per HD18). For patients with negative PET2, we suggest an end of treatment CT scan, whereas for those with a positive PET2, we suggest an end of treatment PET-CT and radiotherapy to residual areas of FDG avidity. We would suggest discussion of radiotherapy for patients with initial disease bulk, especially for those treated with ABVD. In patients over the age of 60 , we would suggest avoiding escBEACOPP and escBEACOPPDac and instead treating with ABVD/ AVD, after careful assessment of frailty and co-morbidities. If ABVD is used, we suggest omitting the bleomycin after cycle 2 irrespective of a PET2 result. For young patients planning a family in the future, especially women, we suggest particular discussion of the relative impact of ABVD versus escBEACOPP/ escBEACOPDac on fertility and also of fertility preservation options.

\section{Authors' conflicts of interest}

GP Collins: Received honoraria and speaker fees from Takeda, Roche, Gilead, BMS, Pfizer.

Celleron; Received research funding from Celgene, Amgen, Pfizer and BMS

CAT Hildyard and SM Vora have no conflicts of interest

\section{Sources of support/ funding}

\section{N/A}

\section{References}

1. Diehl V, Franklin J, Pfreundschuh M, Lathan B, Paulus U, et al. (2003) Standard and increased-dose BEACOPP chemotherapy compared with COPP-ABVD for advanced Hodgkin's disease. $N$ Engl J Med 348: 2386-2395. [Crossref]

2. Engert A, Diehl V, Franklin J, Lohri A, Dörken B, et al. (2009) Escalated-dose BEACOPP in the treatment of patients with advanced-stage Hodgkin's lymphoma: 10 years of follow-up of the GHSG HD9 study. J Clin Oncol 27: 4548-4554. [Crossref]

3. Viviani S, Zinzani PL, Rambaldi A, Brusamolino E, Levis A, et al. (2011) ABVD versus BEACOPP for Hodgkin's lymphoma when high-dose salvage is planned. $N$ Engl J Med 365: 203-212. [Crossref]

4. Carde P (2016) Eight Cycles of ABVD Versus Four Cycles of BEACOPPescalated Plus Four Cycles of BEACOPPbaseline in Stage III to IV, International Prognostic Score $>/=3$, High-Risk Hodgkin Lymphoma: First Results of the Phase III EORTC 20012 Intergroup Trial. J Clin Oncol 34: 2028-2036.

5. Bauer K, Skoetz N, Monsef I, Engert A, Brillant C (2011) Comparison of chemotherapy including escalated BEACOPP versus chemotherapy including ABVD for patients with early unfavourable or advanced stage Hodgkin lymphoma. Cochrane Database Syst Rev: CD007941. [Crossref]

6. Skoetz N, Will A, Monsef I, Brillant C, Engert A, et al. (2017) Comparison of first-line chemotherapy including escalated BEACOPP versus chemotherapy including ABVD for people with early unfavourable or advanced stage Hodgkin lymphoma. Cochrane Database Syst Rev p. 5. 
7. von Tresckow B, Plütschow A, Fuchs M, Klimm B, Markova J, et al. (2012) Doseintensification in early unfavorable Hodgkin's lymphoma: final analysis of the German Hodgkin Study Group HD14 trial. J Clin Oncol 30: 907-913.

8. Engert A, Haverkamp H, Kobe C, Markova J, Renner C, et al. (2012) Reduced-intensity chemotherapy and PET-guided radiotherapy in patients with advanced stage Hodgkin's lymphoma (HD15 trial): a randomised, open-label, phase 3 non-inferiority trial. Lancet 379: 1791-1799.

9. Anderson RA, Remedios R, Kirkwood AA, Patrick P, Stevens L, et al. (2018) Determinants of ovarian function after response-adapted therapy in patients with advanced Hodgkin's lymphoma (RATHL): a secondary analysis of a randomised phase 3 trial. Lancet Oncol 19: 1328-1337.

10. Sieniawski M, Reineke T, Josting A, Nogova L, Behringer K, et al. (2008) Assessment of male fertility in patients with Hodgkin's lymphoma treated in the German Hodgkin Study Group (GHSG) clinical trials. Ann Oncol 19: 1795-1801.

11. Zinzani PL, Tani M, Fanti S, Alinari L, Musuraca G, Marchi E, et al. (2006) Early positron emission tomography (PET) restaging: a predictive final response in Hodgkin's disease patients. Ann Oncol 17: 1296-1300.

12. Johnson P, Trotman J, Federico M (2016) Interim PET-CT Scan in Advanced Hodgkin's Lymphoma. N Engl J Med 375: 999-1000. [Crossref]

13. Borchmann P, Haverkamp H, Lohri A, Mey U, Kreissl S, et al. (2017) Progressionfree survival of early interim PET-positive patients with advanced stage Hodgkin's lymphoma treated with BEACOPPescalated alone or in combination with rituximab (HD18): an open-label, international, randomised phase 3 study by the German Hodgkin Study Group. Lancet Oncol 18: 454-463. [Crossref]

14. Casasnovas (2018) Randomized phase III study comparing an early PET driven treatment de-escalation to a not PET-monitored strategy in patients with advanced stages Hodgkin lymphoma: Final analysis of the AHL2011 LYSA study. Journal of Clinical Oncology 36: 7503-7503.

15. Kobe C, Goergen H, Baues C, Kuhnert G, Voltin CA, et al. (2018) Outcome-based interpretation of early interim PET in advanced-stage Hodgkin lymphoma. Blood 132: 2273-2279.

16. Trotman, J (2017) RESPONSE-ADJUSTED THERAPY FOR ADVANCED HODGKIN LYMPHOMA (RATHL) TRIAL: LONGER FOLLOW UP CONFIRMS EFFICACY OF DE-ESCALATION AFTER a NEGATIVE INTERIM PET SCAN (CRUK/07/033). Hematological Oncology 35: 65-67.

17. Behringer K, Breuer K, Reineke T, May M, Nogova L, et al. (2005) Secondary amenorrhea after Hodgkin's lymphoma is influenced by age at treatment, stage of disease, chemotherapy regimen, and the use of oral contraceptives during therapy: a report from the German Hodgkin's Lymphoma Study Group. J Clin Oncol 30: $7555-$ 7564 .

18. Mauz-Körholz C, Hasenclever D, Dörffel W, Ruschke K, Pelz T, et al. (2010) Procarbazine-free OEPA-COPDAC chemotherapy in boys and standard OPPA-COPP in girls have comparable effectiveness in pediatric Hodgkin's lymphoma: the GPOHHD-2002 study. J Clin Oncol 28: 3680-3686. [Crossref]

19. Johnson PW, Sydes MR, Hancock BW, Cullen M, Radford JA (2010) Consolidation radiotherapy in patients with advanced Hodgkin's lymphoma: survival data from the UKLG LY09 randomized controlled trial (ISRCTN97144519). J Clin Oncol 28: 33523359.

20. Bates JE, Dhakal S, Mazloom A, Casulo C, Constine LS, et al. (2017) Benefit from the inclusion of radiation therapy in the treatment of patients with stage III classical Hodgkin lymphoma: A propensity matched analysis of the Surveillance, Epidemiology, and End Results database. Radiother Oncol 124: 325-330.

21. Connors JM, Radford JA (2018) Brentuximab Vedotin for Stage III or IV Hodgkin's Lymphoma. N Engl J Med 378: 1560-1561.
22. Rassidakis GZ, Medeiros LJ, Viviani S, Bonfante V, Nadali GP, et al. (2002) CD20 expression in Hodgkin and Reed-Sternberg cells of classical Hodgkin's disease: associations with presenting features and clinical outcome. J Clin Oncol 20: 1278-1287.

23. Strati P (2018) Rituximab with ABVD vs ABVD for advanced stage high-risk classical Hodgkin lymphoma: a randomized phase II study. Haematologica.

24. Armand P, Engert A, Younes A, Fanale M, Santoro A, et al. (2018) Nivolumab for Relapsed/Refractory Classic Hodgkin Lymphoma After Failure of Autologous Hematopoietic Cell Transplantation: Extended Follow-Up of the Multicohort SingleArm Phase II CheckMate 205 Trial. J Clin Oncol 36: 1428-1439.

25. Younes A, Santoro A, Shipp M, Zinzani PL, Timmerman JM, et al. (2016) Nivolumab for classical Hodgkin's lymphoma after failure of both autologous stem-cell transplantation and brentuximab vedotin: a multicentre, multicohort, single-arm phase 2 trial. Lancet Oncol 17: 1283-1294.

26. Roemer MG, Advani RH, Ligon AH, Natkunam Y, Redd RA, et al. (2016) PD-L1 and PD-L2 Genetic Alterations Define Classical Hodgkin Lymphoma and Predict Outcome. J Clin Oncol 34: 2690-2697.

27. Ramchandren R (2018) Checkmate 205 cohort D: a phase 2 trial of nivolumab for newly diagnosed advanced-stage classical Hodgkin lymphoma. In 23rd Congress of the European Hematology Association, Stockholm, Sweden.

28. Engert A, Ballova V, Haverkamp H, Pfistner B, Josting A, et al. (2005) Hodgkin's lymphoma in elderly patients: a comprehensive retrospective analysis from the German Hodgkin's Study Group. J Clin Oncol 23: 5052-5060.

29. Borchmann P, Haverkamp H, Diehl V, Cerny T, Markova J, et al. (2011) Eight cycles of escalated-dose BEACOPP compared with four cycles of escalated-dose BEACOPP followed by four cycles of baseline-dose BEACOPP with or without radiotherapy in patients with advanced-stage hodgkin's lymphoma: final analysis of the HD12 trial of the German Hodgkin Study Group. J Clin Oncol 29: 4234-42.

30. Boll B, Görgen H, Fuchs M, Pluetschow A, Eich HT, et al. (2013) ABVD in olde patients with early-stage Hodgkin lymphoma treated within the German Hodgkin Study Group HD10 and HD11 trials. J Clin Oncol 31: 1522-1529.

31. Martin WG, Ristow KM, Habermann TM, Colgan JP, Witzig TE, et al. (2005) Bleomycin pulmonary toxicity has a negative impact on the outcome of patients with Hodgkin's lymphoma. J Clin Oncol 23: 7614-7620. [Crossref]

32. Böll B, Goergen H, Behringer K, Bröckelmann PJ, Hitz F, et al. (2016) Bleomycin in older early-stage favorable Hodgkin lymphoma patients: analysis of the German Hodgkin Study Group (GHSG) HD10 and HD13 trials. Blood 127: 2189-2192.

33. Kolstad A, Nome O, Delabie J, Lauritzsen GF, Fossa A, et al. (2007) Standard CHOP21 as first line therapy for elderly patients with Hodgkin's lymphoma. Leuk Lymphoma 48: 570-576. [Crossref]

34. Proctor SJ, Wilkinson J, Jones G, Watson GC, Lucraft HH, et al. (2012) Evaluation of treatment outcome in 175 patients with Hodgkin lymphoma aged 60 years or over: the SHIELD study. Blood 119: 6005-6015. [Crossref]

35. Boll B (2011) Phase 2 study of PVAG (prednisone, vinblastine, doxorubicin, gemcitabine) in elderly patients with early unfavorable or advanced stage Hodgkin lymphoma. Blood 118: 6292-6298.

36. Holte H, Mella O, Wist E, Telhaug R, Hannisdal E, et al. (1996) ChlVPP is as effective as alternating ChlVPP/ABOD in advanced stage Hodgkin's disease. Acta Oncol 35 Suppl 8: 73-80. [Crossref]

37. Holkova B, Goldschmidt J, Chen R, Olsen G, et al. (2015) Phase 2 study of frontline brentuximab vedotin monotherapy in Hodgkin lymphoma patients aged 60 years and older. Blood 126: 2798-2804.

38. A Phase II Study to Determine Pembrolizumab as Frontline Treatment of Patients With Hodgkin Lymphoma (PLIMATH). ClinicalTrials.gov.

Copyright: $\ 2018$ Hildyard CAT. This is an open-access article distributed under the terms of the Creative Commons Attribution License, which permits unrestricted use, distribution, and reproduction in any medium, provided the original author and source are credited. 